\title{
Title: Heart rate variability and depressive symptoms: a cross-lagged analysis over a 10 year period in the Whitehall II study
}

\author{
Authors' names: Vera K. Jandackova, $\mathrm{PhD}^{\mathrm{ab} *}$; Annie Britton, $\mathrm{PhD}^{\mathrm{b}}$; \\ Marek Malik, PhD MDc; Andrew Steptoe, DPhil ${ }^{\mathrm{b}}$
}

\begin{abstract}
Affiliations:
aDepartment of Epidemiology and Public Health, University of Ostrava, CZ;

${ }^{\mathrm{b}}$ Research Department of Epidemiology and Public Health, University College London, UK;

${ }^{\mathrm{c}}$ National Heart and Lung Institute, Imperial College, London, UK
\end{abstract}

Corresponding author and contact details: Vera K. Jandackova, Department of Epidemiology and Public Health, Faculty of Medicine, University of Ostrava, Syllabova 19, 70300 Ostrava, Czech Republic, email: vera.jandackova@osu.cz, tel: +420605782643

Short title: Prospective associations between HRV and depression

Word count: 4957 (abstract 241) 


\begin{abstract}
Background: People with depression tend to have lower heart rate variability (HRV), but the temporal sequence is poorly understood. In a sample of the general population, we prospectively examined whether HRV measures predict subsequent depressive symptoms or whether depressive symptoms predict subsequent levels of HRV.
\end{abstract}

Method: Data from the fifth (1997-1999) and ninth (2007-2009) phases of the UK Whitehall II longitudinal population-based cohort study were analysed with an average follow-up of 10.5 years. The sample size for the prospective analysis depended on the analysis and ranged from 2334 (644 women) to 2276 (602 women). HRV measures during 5 minutes of supine rest were obtained. Depressive symptoms were evaluated by 4 cognitive symptoms of depression from the General Health Questionnaire.

Results: At follow-up assessment, depressive symptoms were inversely associated with HRV measures independently of antidepressant medication use in men but not in women. Prospectively, higher baseline heart rate and higher HRV measures were associated with a lower likelihood of incident depressive symptoms at follow-up in men without depressive symptoms at baseline. Similar but statistically insignificant associations were found in women. Adjustments for known confounders including socio-demographic and lifestyle factors, cardio-metabolic conditions or medication did not change the predictive effect of HRV on incident depressive symptoms at follow-up. Depressive symptoms at baseline were not associated with heart rate or HRV at follow-up in either sex.

Conclusions: These findings are consistent with an etiologic role of the autonomic nervous system in the depression onset.

Keywords: depressive symptoms, autonomic, heart rate variability, longitudinal cohort study, etiological significance 


\section{Introduction}

Epidemiological and clinical research suggests that depression is associated with a host of cardiovascular conditions (Hare et al. 2014). Depression is common in cardiac patients, while depressive symptoms also predict future cardiovascular disease (CVD) (Nicholson et al. 2006; Hare et al. 2014). A variety of mechanisms underlying this association have been postulated (Grippo \& Johnson 2009; Nemeroff \& Goldschmidt-Clermont 2012).

Disturbed autonomic nervous system (ANS), including decreased parasympathetic and/or increased sympathetic tone, may be one pathway linking depression and negative cardiac outcomes (Stapelberg et al. 2012). Heart rate variability (HRV) is non-invasive technique estimating the ANS characteristics (Task Force 1996). Low HRV indicative of diminished vagal cardiovascular modulations is a known predictor of cardiac morbidity and mortality, and an indicator of stress vulnerability and low capacity for parasympathetic inhibition of autonomic arousal in emotional regulation (Task Force 1996; Thayer et al. 2012).

A number of epidemiological and clinical studies have shown depression to be associated with unfavourable HRV indices, notably reduced cardiac vagal modulation (Rottenberg 2007; Licht et al. 2008, 2010; Kemp et al. 2010; Dauphinot et al. 2012; Brunoni et al. 2013). For example, a meta-analysis of clinical studies demonstrated that relative to controls, physically healthy un-medicated patients with major depressive disorder displayed reduced vagal HRV indices (Kemp et al. 2010). In a more recent double-blind, randomized, placebo-controlled trial, moderately to severely depressed un-medicated patients with low cardiovascular risk profile had lower HRV compared to controls (Brunoni et al. 2013). There is epidemiological evidence that the depression/HRV relationship is partly or fully driven by use of antidepressants (Licht et al. 2008, 2010; Kemp et al. 2014; O’Regan et al. 2014). 
However, this does not apply to un-medicated participants (Kemp et al. 2010; Brunoni et al. 2013).

Although the association between HRV and depression has been studied extensively, the directionality of the HRV-depression relationship received less attention. Possible mechanisms exist for a bidirectional relationship. On the one hand, chronic exposure to stressful experiences and prolonged negative emotions have been shown to lead to increased sympathetic and reduced parasympathetic modulation (Grippo \& Johnson 2009) suggesting that depression may precede low HRV. On the other hand, other evidence supports the possibility that low HRV precedes depression. Namely, (1) reduced modulation of vagal activity and HRV leads to a variety of conditions associated with increased allostasis and poor health (Thayer \& Sternberg 2006); (2) vagal stimulation, associated with HRV increase (Zhang et al. 2009) improves mood and reduces depressive symptoms severity both in humans and animals (Vonck et al. 2014); (3) rats with complete disconnection of abdominal vagal afferents displayed reduced innate negative emotional behaviour compared to controls (Klarer et al. 2014); and (4) reduced vagal HRV indices has been shown to predict a more pernicious course of depression (Chambers \& Allen 2002; de Guevara et al. 2004). Rottenberg (2007) has argued that prospective study designs should be used to test the potential etiological role of the ANS in depression in individuals with no history of depression.

The Whitehall II study is a large longitudinal population-based cohort study in which short-term HRV and cognitive symptoms of depression were measured repeatedly over a 10 year interval. This has enabled us to prospectively examine whether decreased HRV predicts subsequent incident depressive symptoms in non-depressed individuals, and whether depressive symptoms predict subsequent HRV reductions. Additionally, we also evaluated to 
what extent potential longitudinal associations were influenced by covariates including sociodemographic and lifestyle factors, cardio-metabolic condition and medication use.

\section{Methods}

\section{Study population}

The Whitehall II study is an ongoing cohort study of subjects initially employed by the British civil service originally set up to explore the degree and causes of the social gradient in morbidity and mortality. The target population was all London-based office staff, aged 35-55 years. A total of 10,308 subjects $(3,413$ women, response rate of $73 \%)$, were initially recruited between 1985 and 1988 (Marmot \& Brunner 2005). After the baseline examination, follow-up medical examinations have taken place approximately every 5 years. HRV recordings were made at the fifth (1997-1999), seventh (2002-2004) and ninth (2007-2009) data collection phases. The University College London ethics committee approved the study and participants gave informed consent. Whitehall II data, protocols, and other metadata are available to bona fide researchers for research purposes (data sharing policy is available at http://www.ucl.ac.uk/whitehallII/data-sharing).

The present study included participants with data on cognitive symptoms of depression and HRV at phase 5 (1997-1999) or phase 9 (2007-2009). Mean follow-up was 10.5 years (range $8.9-12.3$ ). Phase 5 is used as the baseline and phase 9 as the follow-up. The number of participants who had data on cognitive symptoms of depression and HRV was 949 women and 2268 men at baseline, and 1293 women and 3451 men at follow-up. The sample size for the prospective analysis ranged from 2334 (644 women) to 2276 (602 women) dependent on the completeness of covariate data.

\section{HRV measurements}


Details on the assessment of heart rate and HRV in Whitehall II can be found elsewhere (Hemingway et al. 2005; Britton et al. 2007). Briefly, five minute supine resting 12-lead electrocardiograms (ECG) were obtained after 5 min of rest. At phases 5 and 9, Kardiosis ${ }^{\mathrm{TM}}$ ECG acquisition module (Tepa, Inc., Turkey) and Getemed ECG recorder (Getemed Teltow, Germany) were used, respectively. The sampling frequencies and outputs of the different devices were comparable. Using an automatic algorithm (Acar et al. 2000), normal sinusrhythm QRS complexes suited for a reliable HRV analysis were identified. Although in shortterm recordings obtained at standardized conditions, spectral HRV analysis is the preferred method (Task Force 1996), in this study HRV was analysed in both time- and frequencydomain. Time-domain measures included the standard deviation (SD) of all normal-to-normal RR intervals (SDNN) and root mean square of successive differences of normal-to-normal RR intervals (RMSSD). Frequency-domain components were computed using a Blackman-Tukey algorithm. The low frequency (LF-HRV), 0.04 to $0.15 \mathrm{~Hz}$, and high frequency (HF-HRV), 0.15 to $0.4 \mathrm{~Hz}$, spectral power was obtained. The RMSSD and HF-HRV reflect parasympathetic modulation. The interpretation of LF-HRV is controversial but most evidence suggest that it reflects both parasympathetic and sympathetic modulations. Under particular circumstances, it may be influenced by different regulatory mechanisms including, among others, respiration and baroreflex control (Task Force 1996; Bernardi et al. 2000; Lahiri et al. 2008; Goldstein et al. 2011; Reyes del Paso et al. 2013).

\section{Depressive symptoms}

Symptoms of depression were measured using a 4-item scale derived from the 30-item General Health Questionnaire (GHQ) (Goldberg \& Hillier 1979). The General Health Questionnaire is used to detect minor psychiatric disorders including depressive disorder, suitable for use in general population samples (GHQ) (Goldberg 1972). The 30-item GHQ 
was validated within the Whitehall II Study (Stansfeld et al. 1998) based on principal components factor analysis and a comparison with the 7-item severe depression subscale from the 28-item GHQ. The depression symptom score includes the following four items: "been thinking of yourself as a worthless", "felt that life is entirely hopeless", "felt that life isn't worth living", and "found at times you couldn't do anything because your nerves were too bad" (Cronbach's $\alpha=0.88)$. Responses to these items were on a four-point Likert scale $(0=$ "not at all", 1 = "no more than usual", 2 = "rather more than usual", and $3=$ "much more than usual") giving a range of 0 to 12. A sum score was calculated and, as previously, a total score of 4 or more was used to define cognitively based symptoms of depression (Stansfeld et al. 1998). The test-retest reliability was $r=0.78$ in a sub-sample of 286 participants who repeated the GHQ within 1 month (Stansfeld et al. 2003). The respondents were considered as having a depressive episode if they scored $\geq 4$ on the GHQ depression subscale or reported the use of prescribed antidepressant medication (Stansfeld et al. 2003).

\section{Covariates}

Relevant confounders that have been previously shown to be associated with HRV and depression (Huikuri et al. 1998; Rennie et al. 2003; Hemingway et al. 2005; Marmot \& Brunner 2005; Schroeder et al. 2005; Britton et al. 2007) were included as covariates and were obtained from phase 5 unless otherwise stated. Socio-demographic characteristics included age (mean), ethnicity (white vs. other) and civil service employment grade. Lifestyle factors included leisure-time physical activity, alcohol consumption and smoking habits, and were assessed by self-completed questionnaires. Participants were asked how often they took part in moderate and vigorous exercise such as walking, cycling, sports, gardening, housework, and home maintenance. Those participants undertaking less than 1hour per week of vigorous physical activity or less than 2.5 hours per week of moderate physical activity did not meet WHO recommendations (2010) and were defined as exercising little or none (Sabia 
et al. 2009). Alcohol consumption in the past week was expressed in units of alcohol (1 $\mathrm{U}=8$ $\mathrm{g}$ ), and $\geq 14 \mathrm{U} /$ week in women and $\geq 21 \mathrm{U} /$ week in men were categorized as high consumption. Smoking habits were categorized as non/ex/current smoker. Cardio-metabolic conditions were assessed as the presence of any of the following chronic diseases or healthrelated conditions: diagnosed CHD including heart failure, stroke, diabetes, hypertension (use of hypertensive medication or blood pressure $\geq 140 / 90 \mathrm{mmHg}$ ) and obesity (body mass index, $\mathrm{BMI} \geq 30 \mathrm{~kg} / \mathrm{m} 2$ ). Blood pressure and BMI were assessed according to standard guidelines. Medication use included any prescribed medication within last 14 days. Antidepressant medication use both at phase 5 and 9 was recorded separately as previous studies have shown antidepressant treatment to influence the HRV-depression association (Licht et al. 2008, 2010; O’Regan et al. 2014).

\section{Statistical analysis}

Linear and logistic regressions were used to explore cross-sectional and prospective associations between HRV and depression symptoms. Heart rate and HRV measures were used as continuous variables. Depressive symptoms were used as a continuous and categorical variable for cross-sectional and prospective analyses, respectively. Models were adjusted for age and ethnicity since they are known to influence HRV (Task Force 1996; Hemingway et al. 2005; Britton et al. 2007). Results are presented as odds ratios (OR) or unstandardized regression coefficients $(\beta)$ with $95 \%$ confidence intervals (CIs). The level of statistical significance was set at $\mathrm{p} \leq 0.05$. Intentionally, we have not corrected the $\mathrm{p}$-values for multiple comparisons (Rothman 1990, 2014; Saville 1990). HRV values were logarithmically transformed prior to analyses to handle their skewed distributions. Depression score was also skewed but none of the transformations (log, square-root, cubic, square, inverse and others) provided normal distribution of data so results are presented in terms of raw depression 
scores, for ease of interpretation. The results of both cross-sectional and prospective analyses were virtually unchanged when depression score was log or square-root transformed (data not shown).

Cross-sectional analyses, undertaken among participants with no antidepressant medication use were assessed by linear regression both at phase 5 and phase 9 . The first prospective model, using binary logistic regression, examined whether HRV at phase 5 (19971999) was associated with incident depressive symptoms at phase 9 (2007-2009), while adjusting for depressive symptoms score at phase 5 (1997-1999). This analysis focused on participants without a depression at phase 5 baseline, i.e. participants with GHQ score $\geq 4$ or those reporting antidepressant medication use at phase 4 (1995-1996) and 5 (1997-1999) were excluded. Cases of incident depressive symptoms at follow-up were defined as participants having GHQ score $\geq 4$ or reporting use of antidepressant medication at phase 9 (2007-2009). In the second prospective model, linear regression explored the association between depressive symptoms at phase 5 (1997-1999) and HRV measures at phase 9 (2007-2009), while adjusting for HRV at phase 5 (1997-1999). Cases of incident depressive symptoms at baseline (phase 5) were defined as participants having GHQ score $\geq 4$ or reporting antidepressant medication use at phase 5 .

Analyses were not constrained to participants with complete data on HRV measures and cognitive symptoms of depression at both phases. To ensure that sample differences did not account for differences in results, models were repeated using the same sample for all analyses. This had little effect on the pattern of associations, so results are presented using all the available data for each analysis.

In order to assess the predictive ability of HRV measures we used receiver operator characteristic (ROC) analysis to generate area under the ROC curve (AUC). The ROC curves plot the positive fraction, or sensitivity against the false positive fraction (1-specificity) by 
varying the threshold value for the test. The ROC curve indicates the probability of a true positive result as a function of the probability of a false positive result for all possible threshold values. The AUC is a quantitative measure of selectivity: 0.5 indicates that the test results are no better than those obtained by chance, whereas an area of 1.0 indicates a perfectly sensitive and specific test. The contribution of the covariates to the association between HRV and symptoms of depression was explored in logistic regression models. To the basic model adjusted for age, sex and ethnicity each of the following sets of factors were individually included: employment grade, lifestyle factors, cardio-metabolic condition and medication use. Subsequently, a model adjusted for all covariates was used. All analyses were computed in STATA (version 12; StataCorp LP, College Station, TX, USA).

\section{Results}

Of the 10,308 participants at phase 1 (1985-1988), 306 died before the start of HRV data collection at phase 5 (1997-1999). Table 1 presents the descriptive characteristics of participants with data on both depressive symptoms and HRV either at phase 5 baseline or phase 9 follow-up. At both phases, there were more males $(70.5 \%$ and $72.6 \%)$ and the majority of participants were of white origin. Average levels of depressive symptoms and heart rate, RMSSD and HF-HRV were higher $(\mathrm{p} \leq 0.005)$ and LF-HRV lower $(\mathrm{p} \leq 0.001)$ in women than in men. Women included a higher proportion of participants from ethnic minorities and were more likely to be from the lower employment grades. On average, twice as many women than men were taking antidepressant medication. Compared with phase 5, at phase 9 there was about one third higher proportion of women and men taking antidepressant medication and consequently lower average levels of depressive symptoms.

\section{Insert Table 1 here}


Table 2 shows the cross-sectional associations between HRV measures (but not HR and RMSSD) and depressive symptoms at baseline (phase 5) and follow-up (phase 9) in participants not using antidepressant medication. In these subjects, depressive symptoms score was not significantly associated with heart rate or HRV at baseline. In the crosssectional analyses at follow-up, higher depressive symptoms score was significantly associated with lower SDNN $(\beta=-0.01 ; p=0.04)$, LF-HRV $(\beta=-0.03 ; p=0.02)$ and HFHRV ( $\beta=-0.04 ; p=0.04)$ among men, but not among women. At both phases, there were no significant associations between depressive symptoms score and HR or RMSSD in both sexes.

\section{Insert Table 2 here}

Table 3 and Figure 1 present the results from the prospective logistic analysis estimating associations between HRV at baseline (phase 5) and depressive symptoms at follow-up (phase 9). Results indicated that among men without a depressive episode at phase 4 (1995-1996) and 5 (1997-1999), HRV at baseline (phase 5) predicted incident depressive symptoms at follow-up (phase 9). Specifically, lower heart rate and higher SDNN, RMSSD, LF-HRV and HF-HRV were associated with lower likelihood of depressive symptoms (Heart rate: OR = 1.02, 95\% CI. 1.01-104, $\mathrm{p}=0.011 ; \mathrm{SDNN}: \mathrm{OR}=0.57,95 \%$ CI. $0.36-0.89, \mathrm{p}=0.013$; RMSSD: OR $=0.65,95 \%$ CI. 0.46-0.92, $\mathrm{p}=0.016 ; \mathrm{LF}-\mathrm{HRV}: \mathrm{OR}=0.77,95 \%$ CI. 0.63-0.94, $\mathrm{p}=0.010 ;$ HF-HRV: OR $=0.81,95 \%$ CI. 0.67-0.98, $\mathrm{p}=0.031)$. For example, for men, the odds of elevated depressive symptoms or antidepressant medication use at follow-up decreased by $35 \%$ for every unit increase in log-transformed RMSSD or by $19 \%$ for every unit increase in log-transformed HF-HRV. Age-adjusted arithmetic- and geometric-means of 
HR and HRV measures at phase 5 for those men who developed depressive symptoms at follow-up ( $\mathrm{n}=106)$ compared to those who did not $(\mathrm{n}=1584)$ are as follows: for HR $71.7 \mathrm{bpm}$ vs. 68.4 bpm; for SDNN 30.9ms ${ }^{2}$ vs $34.8 \mathrm{~ms}^{2}$; for LF-HRV $259.5 \mathrm{~ms}^{2}$ vs. $338.1 \mathrm{~ms}^{2}$; for HFHRV $95.6 \mathrm{~ms}^{2}$ vs. $121 \mathrm{~ms}^{2}$; for RMSSD $17.1 \mathrm{~ms}^{2}$ vs. $20 \mathrm{~ms}^{2}$. The receiver operator curve analysis yielded AUC of 0.72 (95\% CI. 0.67-0.78) for SDNN and 0.73 (95\% CI. 0.67-0.79) for HR, LF-HRV, HF-HRV and RMSSD. For women, the predictive effect of heart rate or HRV in incident depressive symptoms did not reach the statistically significant level $(\mathrm{p} \geq$ 0.062), although the HRV associations were similar to those in men (AUC: $0.67-0.69,95 \%$ CI. 0.60-0.76).

\section{Insert Table 3 here}

Table 4 presents the results from the adjusted prospective logistic analyses assessing the contribution of the five sets of covariates to the association between HRV at baseline and cognitive symptoms of depression at follow-up in men. None of the covariates, individually or in combination, accounted for more than a relatively small part of the association $(4.95 \%$ for SDNN, $1.95 \%$ for HF-HRV and 3.76\% RMSSD in combination of all covariates). In women, covariate adjustment did not alter the non-significant association between HRV at baseline and depressive symptoms at follow-up (data not shown).

\section{Insert Table 4 here}

The examination of cognitive symptoms of depression at baseline (phase 5) as predictors of heart rate and HRV measures at follow-up (phase 9) did not reveal any significant association in either sex ( $p>0.348)$ (data not shown). 


\section{Discussion}

Based on a large community sample, our investigation suggests that cognitive symptoms of depression are associated with HRV independently of antidepressant medication use, in both cross-sectional and longitudinal associations. Over a 10-years period, we found that in individuals without depressive symptoms, lower heart rate and higher SDNN, RMSSD, LFHRV and HF-HRV predicted subsequent incident depressive symptoms. This association was independent of socio-demographic and lifestyle factors, cardio-metabolic condition and/or medication use and baseline cognitive symptoms of depression. Depressive symptoms did not predict subsequent levels of heart rate or HRV. Results suggest that disturbances of cardiac autonomic status, particularly low vagal modulation, precede depressive symptoms.

The findings in the cross-sectional analyses at phase 9 are consistent with previous clinical and epidemiological studies showing an inverse association of HRV with depressive symptoms or major depressive disorder independently of antidepressant treatment (Kemp et al. 2010; Dauphinot et al. 2012; Brunoni et al. 2013). Although the significant crosssectional association was observed only at phase 9, the cross-sectional effect is nevertheless present, suggesting that antidepressant use does not fully drive the relationship between HRV and depression as some authors report in other studies (Licht et al. 2008, 2010; Kemp et al. 2014; O'Regan et al. 2014). We believe that the cross-sectional association at phase 5 was not significant due to smaller sample size at phase 5 compared with phase 9 . As seen in the Table 2, regression coefficients for some HRV measures (e.g. SDNN and LF-HRV in men) did not change much from phase 5 to phase 9 , but they were significant in phase 9 only. In order to further explore the cross-sectional association between HRV and depressive symptoms we conducted an analysis using data from phase 7. Results indicated similar size of the regression coefficients between depressive symptoms and HRV measures and significant associations in 
men (data not shown). This supports the presence of the overall cross-sectional association between HRV and depressive symptoms independently of antidepressant treatment.

A number of previous studies have tested whether lower vagal HRV indices among depressed individuals predict a more pernicious course of disorder (Chambers \& Allen 2002; de Guevara et al. 2004). To our knowledge, the predictive effect of HRV on incident depression in a large sample of general population without depressive symptoms at baseline has not been investigated before. The cognitive symptoms of depression can be regarded as a part of the prodromal phase of major depressive disorder and therefore can be considered as a significant risk indicator of major depressive disorder (Cuijpers \& Smit 2004). Our results suggest that low HRV and vagal modulation, as reflected by decreased RMSSD and HFHRV, play an etiologic role in incident depression rather than contributing to the later stages of depression development among men. As seen in Table 3 the odds of depressive symptoms at follow-up decreased by $43 \%$ for every unit increase in log-transformed SDNN and by $35 \%$ and $19 \%$ for every unit increase in log-transformed RMSSD and HF-HRV. Additionally, ROC analysis revealed a high sensitivity and specificity (0.72-0.73) of HR and HRV measures for the prediction of incidence cognitive symptoms of depression in men. Findings indicate that the contribution of ANS disturbance to depressive symptoms is quite large.

The findings on the predictive effect of HRV in depression generation in men accords with the study by Gimeno et al. (2009). Over a period of 12 years within Whitehall II population, inflammatory markers were shown to precede subsequent cognitive symptoms of depression while depressive symptoms did not precede subsequent average levels of inflammatory markers (Gimeno et al. 2009). As the ANS plays a homeostatic role in relation to the inflammatory response (Tracey 2002), and lower HRV has been shown to predict inflammation (Jarczok et al. 2014), our results are complementary to earlier work (Gimeno et al. 2009). The predictive effects of HRV, and inflammation respectively, on future depressive 
symptoms were independent of potential covariates and stronger in men than in women. A possible alternative interpretation accounting for the lack of predictive association between depressive symptoms and subsequent physiological functioning (as indexed by average levels of HRV) in our study, may be the long period of follow-up and narrow scope of the depression measure. Assessment of neuro-vegetative symptoms, which might more readily be expected to be associated with physiological disturbances, may have produced stronger crosssectional association and potentially evidence of bidirectional association between HRV and depression.

Our findings of etiological significance of HRV in depression generation is in agreement with polyvagal theory and neuro-visceral integration model (Porges 1995; Thayer \& Lane 2000). According to these theories the affective and social behaviour in mammals is dependent on the ability to regulate visceral homeostasis, including control of heart, mediated by vagal signalling (Porges 1995; Rottenberg 2007; Thayer et al. 2012). Findings of neuroimaging studies (Thayer et al. 2012), have indeed shown low HRV and vagal modulation to be associated with number of brain regions, including prefrontal cortex and amygdala, that are involved in emotional dysregulation. Insufficient vagal functioning, may therefore have a direct impact on emotion and social communication, which can in turn manifest as cognitive symptoms of depression or major depressive disorder (Rottenberg 2007). Therefore the wellknown association between depressive symptoms and cardiac health may be due in part to disturbed ANS and low vagal modulation, rather than depression per se. Based on results of our and previous studies, low HRV and vagal modulation may be risk factors for both depression and CHD. Vagal pathways could therefore be intervention targets. For instance, it has been shown that enhancing vagal cardiac modulation by physical activity or vagal stimulation, have favorable effects on HRV, emotions or cardiac health (Soares-Miranda et al. 2014; Vonck et al. 2014). 
It is unclear why the prospective association was significant only among men and not among women even though it was in the expected direction. As the number of men was nearly three times larger than of women, it may be possible that increasing a sample size in women would lead to narrower confidence intervals and probably to significant HRV-depression association. Another possible explanation is that differences in the neural control of the heart and in achieving homeostasis may have impacted our observations (Smetana \& Malik 2013). On average women have reduced sympathetic and increased parasympathetic modulation of heart rate relative to men as well as different biological ways of responding to stress (Hassan et al. 2008). Furthermore depression has been shown to manifest differently in men and women; relative to men, women show more somatic symptoms of depression (Silverstein et al. 2013). It is possible that we were not able to detect depression in some women due the scale used to assess depression.

In our data the associations between HRV and depressive symptoms were not explained by potential covariates. By adjusting for cardio-metabolic condition (the presence of diagnosed CHD, stroke, diabetes, hypertension and/or obesity) we were able to control for the possibility that our observed associations were due to confounding by physical illness and pre-existing illness at baseline. These adjustments explained a maximum of $1.5 \%$ of the HRVdepression association. Similarly medication use within last 2 weeks as well as lifestyle factors and SES had little effect on the association. After simultaneous adjustments for all mentioned covariates the association remained virtually unchanged (explained a maximum of $2 \%$ of the association).

A key strength of our investigation is the use of longitudinal data with two measurements of HRV assessed over a decade in a non-clinical setting. Repeated measures of short-term HRV allow to attribute HRV to parasympathetic or/and sympathetic modulation and generally to cardiac autonomic status. Limitations include that the GHQ depression scale 
used is only a part of the seven item depression scale of the 28-item GHQ. GHQ depression is also best considered to be a measure of depressive symptoms, and should not be equated with clinically diagnosed depression (Stansfeld et al. 1995). It is essentially a measure of selfreported depressive symptoms and although reliable, it does not indicate the severity of depression. There is also the issue that we measured depressive symptoms at a single point in time 10 years after the original assessment. Since depression is episodic, it could be that we have not picked up depressive problems that occurred at other times during this period. We also cannot rule out the contribution of device effects as different recording equipment was used to measure HRV at each phase. However the HRV protocols were consistent across all data collection phases. We also did not use any autonomic provocative maneuver which may limit the HRV data comparisons. Finally, the Whitehall II study is an occupational-based cohort, and is therefore healthier on average than the general population. However, etiological findings from the Whitehall II cohort have been shown to be comparable to other populationbased studies (Batty et al. 2014).

In summary, our findings suggest that low HRV and vagal modulation may underlie deficits in self-regulation in those with depression and may be implicated in the generation of depressive episodes. Improvement in cardiac vagal modulation has potentially important future applications on the treatment of depression.

\section{Acknowledgements and financial support}

The Whitehall II study is supported by grants from the Medical Research Council; British Heart Foundation; Health and Safety Executive; Department of Health; National Heart Lung and Blood Institute (HL36310), US, NIH: National Institute on Aging, US, NIH; Agency for Health Care Policy Research (HS06516); and the JD and CT MacArthur Foundation Research 
Networks on Successful Midlife Development and Socio-economic Status and Health. Vera Jandackova was supported by a SGS23/LF/2015 Award and by a Young Investigator of Moraviansilesian region Award 02679/2014/RRC.

\section{Conflict of interest}

None

\section{Ethical standards}

The authors assert that all procedures contributing to this work comply with the ethical standards of the relevant national and institutional committees on human experimentation and with the Helsinki Declaration of 1975, as revised in 2008. 


\section{References}

Acar B, Savelieva I, Hemingway H, \& Malik M (2000). Automatic ectopic beat elimination in short-term heart rate variability measurement. Computer Methods and Programs in Biomedicine 63, 123-131.

Batty GD, Shipley M, Tabák A, Singh-Manoux A, Brunner E, Britton A, \& Kivimäki M (2014). Generalizability of occupational cohort study findings. Epidemiology (Cambridge, Mass.) 25, 932-3.

Bernardi L, Wdowczyk-Szulc J, Valenti C, Castoldi S, Passino C, Spadacini G, \& Sleight P (2000). Effects of controlled breathing, mental activity and mental stress with or without verbalization on heart rate variability. . UNITED STATES Journal of the American College of Cardiology 35, 1462-1469.

Britton A, Shipley M, Malik M, Hnatkova K, Hemingway H, \& Marmot M (2007). Changes in heart rate and heart rate variability over time in middle-aged men and women in the general population (from the Whitehall II Cohort Study). The American journal of cardiology 100, 524-7.

Brunoni AR, Kemp AH, Dantas EM, Goulart AC, Nunes MA, Boggio PS, Mill JG, Lotufo P a, Fregni F, \& Benseñor IM (2013). Heart rate variability is a trait marker of major depressive disorder: evidence from the sertraline vs. electric current therapy to treat depression clinical study. The international journal of neuropsychopharmacology / official scientific journal of the Collegium Internationale Neuropsychopharmacologicum (CINP) 16, 1937-49.

Cuijpers P, \& Smit F (2004). Subthreshold depression as a risk indicator for major depressive disorder : a systematic review of prospective studies. Acta Psychiatr Scand 109, 325-331.

Dauphinot V, Rouch I, Kossovsky MP, Pichot V, Dorey J-M, Krolak-Salmon P, Laurent B, Roche F, \& Barthélémy J-C (2012). Depressive symptoms and autonomic nervous system dysfunction in an elderly population-based study: the PROOF study. Journal of affective disorders 143, 153-9.

Gimeno D, Kivimäki M, Brunner EJ, Elovainio M, De Vogli R, Steptoe a, Kumari M, Lowe GDO, Rumley a, Marmot MG, \& Ferrie JE (2009). Associations of C-reactive protein and interleukin- 6 with cognitive symptoms of depression: 12-year follow-up of the Whitehall II study. Psychological medicine 39, 413-23.

Goldberg DP (1972). The detection of psychiatric illness by questionnaire; a technique for the identification and assessment of non-psychotic psychiatric illness. London, New York, Oxford University Press.

Goldberg DP, \& Hillier VF (1979). A scaled version of the General Health Questionnaire. . ENGLAND Psychological medicine 9, 139-145. 
Goldstein DS, Bentho O, Park M-Y, \& Sharabi Y (2011). Low-frequency power of heart rate variability is not a measure of cardiac sympathetic tone but may be a measure of modulation of cardiac autonomic outflows by baroreflexes. Experimental physiology 96, 1255-61.

Grippo AJ, \& Johnson AK (2009). Stress, depression and cardiovascular dysregulation: a review of neurobiological mechanisms and the integration of research from preclinical disease models. Stress (Amsterdam, Netherlands) 12, 1-21.

De Guevara MSL, Schauffele SI, Nicola-Siri LC, Fahrer RD, Ortíz-Frágola E, MartínezMartínez J a, Cardinali DP, \& Guinjoan SM (2004). Worsening of depressive symptoms 6 months after an acute coronary event in older adults is associated with impairment of cardiac autonomic function. Journal of affective disorders $\mathbf{8 0}, 257-62$.

Hare DL, Toukhsati SR, Johansson P, \& Jaarsma T (2014). Depression and cardiovascular disease: a clinical review. . England European heart journal 35, 1365-1372.

Hassan M, Li Q, Brumback B, Lucey DG, Bestland M, Eubanks G, Fillingim RB, \& Sheps DS (2008). Comparison of peripheral arterial response to mental stress in men versus women with coronary artery disease. The American journal of cardiology 102, 970-4.

Hemingway H, Shipley M, Brunner E, Britton A, Malik M, \& Marmot M (2005). Does autonomic function link social position to coronary risk? The Whitehall II study. Circulation 111, 3071-7.

Huikuri H V, Ma TH, Airaksinen KEJ, Seppa T, Puukka P, \& Ra IJ (1998). Power-Law Relationship of Heart Rate Variability as a Predictor of Mortality in the Elderly. Circulation 97, 2031-2036.

Chambers AS, \& Allen JJB (2002). Vagal tone as an indicator of treatment response in major depression. Psychophysiology 39, 861-864.

Jarczok MN, Koenig J, Mauss D, Fischer JE, \& Thayer JF (2014). Lower heart rate variability predicts increased level of C-reactive protein 4 years later in healthy, nonsmoking adults. Journal of internal medicine 276, 667-71.

Kemp AH, Brunoni AR, Santos IS, Nunes M a, Dantas EM, Carvalho de Figueiredo R, Pereira AC, Ribeiro ALP, Mill JG, Andreão R V, Thayer JF, Benseñor IM, \& Lotufo P a (2014). Effects of depression, anxiety, comorbidity, and antidepressants on resting-state heart rate and its variability: an ELSA-Brasil cohort baseline study. The American journal of psychiatry 171, 1328-34.

Kemp AH, Quintana DS, Gray M a, Felmingham KL, Brown K, \& Gatt JM (2010). Impact of depression and antidepressant treatment on heart rate variability: a review and meta-analysis. . Elsevier Inc. Biological psychiatry 67, 1067-74.

Klarer M, Arnold M, Günther L, Winter C, Langhans W, \& Meyer U (2014). Gut vagal afferents differentially modulate innate anxiety and learned fear. The Journal of neuroscience : the official journal of the Society for Neuroscience 34, 7067-76. 
Lahiri MK, Kannankeril PJ, \& Goldberger JJ (2008). Assessment of autonomic function in cardiovascular disease: physiological basis and prognostic implications. Journal of the American College of Cardiology 51, 1725-33.

Licht C, de Geus EJC, Zitman FG, Hoogendijk WJG, van Dyck R, \& Penninx BWJH (2008). Association between major depressive disorder and heart rate variability in the Netherlands Study of Depression and Anxiety (NESDA). Arch gen psychiatry 65, 1358-1367.

Licht CMM, de Geus EJC, van Dyck R, \& Penninx BWJH (2010). Longitudinal evidence for unfavorable effects of antidepressants on heart rate variability. . Elsevier Inc. Biological psychiatry 68, 861-8.

Marmot M, \& Brunner E (2005). Cohort Profile: the Whitehall II study. International journal of epidemiology 34, 251-6.

Nemeroff CB, \& Goldschmidt-Clermont PJ (2012). Heartache and heartbreak--the link between depression and cardiovascular disease. . England Nature reviews. Cardiology 9, 526539.

Nicholson A, Kuper H, \& Hemingway H (2006). Depression as an aetiologic and prognostic factor in coronary heart disease: a meta-analysis of 6362 events among 146538 participants in 54 observational studies. European heart journal 27, 2763-74.

O'Regan C, Kenny R a, Cronin H, Finucane C, \& Kearney PM (2014). Antidepressants strongly influence the relationship between depression and heart rate variability: findings from The Irish Longitudinal Study on Ageing (TILDA). Psychological medicine, 1-14.

Pichon A, Nuissier F, \& Chapelot D (2010). Heart rate variability and depressed mood in physical education students: a longitudinal study. . Elsevier B.V. Autonomic neuroscience : basic \& clinical 156, 117-23.

Porges SW (1995). Orienting in a defensive world: mammalian modifications of our evolutionary heritage. A Polyvagal Theory. . UNITED STATES Psychophysiology 32, 301318.

Rennie KL, Hemingway H, Kumari M, Brunner E, Malik M, \& Marmot M (2003). Effects of Moderate and Vigorous Physical Activity on Heart Rate Variability in a British Study of Civil Servants. American Journal of Epidemiology 158, 135-143.

Reyes del Paso G a., Langewitz W, Mulder LJM, van Roon A, \& Duschek S (2013). The utility of low frequency heart rate variability as an index of sympathetic cardiac tone: A review with emphasis on a reanalysis of previous studies. Psychophysiology 50, 477-487.

Rothman KJ (1990). No adjustments are needed for multiple comparisons. UNITED STATES Epidemiology (Cambridge, Mass.) 1, 43-46.

Rothman KJ (2014). Six persistent research misconceptions. Journal of general internal medicine 29, 1060-4. 
Rottenberg J (2007). Cardiac vagal control in depression: a critical analysis. Biological psychology 74, 200-11.

Sabia S, Nabi H, Kivimaki M, Shipley MJ, Marmot MG, \& Singh-Manoux A (2009). Health behaviors from early to late midlife as predictors of cognitive function: The Whitehall II study. American journal of epidemiology 170, 428-37.

Saville D (1990). Multiple Comparison Procedures: The Practical Solution. The American Statistician 44, 174-180.

Schroeder EB, Chambless LE, Liao D, Prineas RJ, Evans GW, Rosamond WD, \& Heiss G (2005). Diabetes, Glucose, Insulin, and Heart Rate Variability. Diabetes Care 28, 668-674.

Silverstein B, Edwards T, Gamma a, Ajdacic-Gross V, Rossler W, \& Angst J (2013). The role played by depression associated with somatic symptomatology in accounting for the gender difference in the prevalence of depression. Social psychiatry and psychiatric epidemiology 48, 257-63.

Smetana P, \& Malik M (2013). Sex differences in cardiac autonomic regulation and in repolarisation electrocardiography. Pflugers Archiv : European journal of physiology

Soares-Miranda L, Sattelmair J, Chaves P, Duncan GE, Siscovick DS, Stein PK, \& Mozaffarian D (2014). Physical activity and heart rate variability in older adults: the Cardiovascular Health Study. Circulation 129, 2100-10.

Stansfeld S a, North FM, White I, \& Marmot MG (1995). Work characteristics and psychiatric disorder in civil servants in London. Journal of Epidemiology \& Community Health 49, 48-53.

Stansfeld SA, Head J, Fuhrer R, Wardle J, \& Cattell V (2003). Social inequalities in depressive symptoms and physical functioning in the Whitehall II study: exploring a common cause explanation

Stansfeld SA, Head J, \& Marmot MG (1998). Explaining social class differences in depression and well-being. . GERMANY Social psychiatry and psychiatric epidemiology 33, $1-9$.

Stapelberg NJ, Hamilton-Craig I, Neumann DL, Shum DHK, \& McConnell H (2012). Mind and heart: heart rate variability in major depressive disorder and coronary heart disease - a review and recommendations. The Australian and New Zealand journal of psychiatry 46, $946-57$.

Task Force of the European Society of Cardiology and The North American Society of Pacing and Electrophysiology (1996). Heart rate variability. Standards of measurement, physiological interpretation, and clinical use. Task Force of the European Society of Cardiology and the North American Society of Pacing and Electrophysiology. European heart journal 17, 354-81.

Thayer JF, Ahs F, Fredrikson M, Sollers JJ, \& Wager TD (2012). A meta-analysis of heart rate variability and neuroimaging studies: implications for heart rate variability as a 
marker of stress and health. . Elsevier Ltd Neuroscience and biobehavioral reviews 36, 74756.

Thayer JF, \& Lane RD (2000). A model of neurovisceral integration in emotion regulation and dysregulation. Journal of affective disorders 61, 201-16.

Thayer JF, \& Sternberg E (2006). Beyond heart rate variability: vagal regulation of allostatic systems. Annals of the New York Academy of Sciences 1088, 361-72.

Vonck K, Raedt R, Naulaerts J, De Vogelaere F, Thiery E, Van Roost D, Aldenkamp B, Miatton M, \& Boon P (2014). Vagus nerve stimulation...25 years later! What do we know about the effects on cognition? . Elsevier Ltd Neuroscience and biobehavioral reviews $\mathbf{4 5}$, 63-71.

Zhang Y, Popovic ZB, Bibevski S, Fakhry I, Sica D a, Van Wagoner DR, \& Mazgalev TN (2009). Chronic vagus nerve stimulation improves autonomic control and attenuates systemic inflammation and heart failure progression in a canine high-rate pacing model. Circulation. Heart failure 2, 692-9. 
Table 1 Correlates of HRV and depressive symptoms at phase 5 (1997-1999) and 9 (20072009) by sex

\begin{tabular}{|c|c|c|c|c|}
\hline \multirow[b]{3}{*}{$\mathrm{n}$} & \multicolumn{2}{|c|}{ Phase 5 (1997-1999) } & \multicolumn{2}{|c|}{ Phase $9(2007-2009)$} \\
\hline & Men & Women & Men & Women \\
\hline & 2,268 & 949 & 3,451 & 1,293 \\
\hline GHQ depressive symptoms score, mean (SD) & $1.00(1.8)$ & $1.25(2.0)$ & $0.66(1.45)$ & $0.89(1.71)$ \\
\hline GHQ depression caseness (GHQ $\geq 4), \mathrm{n}(\%)$ & $283(12.5)$ & $145(15.3)$ & $270(7.5)$ & $133(9.8)$ \\
\hline Heart rate $\mathrm{b} / \mathrm{min}(\mathrm{SE})$ & $68.89(0.22)$ & $70.81(0.34)$ & $66.72(0.19)$ & $68.72(0.31)$ \\
\hline SDNN ms $(\mathrm{SE})^{\mathrm{a}}$ & $34.06(1.01)$ & $32.54(1.01)$ & $30.34(1.01)$ & $29.56(1.01)$ \\
\hline LF-HRV $\mathrm{ms}^{2}(\mathrm{SE})^{\mathrm{a}}$ & $319.68(1.02)$ & $243.98(1.03)$ & $234.75(1.02)$ & $197.29(1.03)$ \\
\hline $\mathrm{HF}-\mathrm{HRV} \mathrm{ms}^{2}(\mathrm{SE})^{\mathrm{a}}$ & $115.29(1.02)$ & $140.21(1.04)$ & $86.42(1.03)$ & $106.25(1.03)$ \\
\hline $\operatorname{RMSSD} \mathrm{ms}(\mathrm{SE})^{\mathrm{a}}$ & $19.56(1.01)$ & $20.91(1.02)$ & $18.34(1.01)$ & $19.27(1.02)$ \\
\hline Age, y, mean (SD) & $55.7(6.1)$ & $56.5(6.1)$ & $65.4(5.8)$ & $65.2(5.7)$ \\
\hline White ethnic origin, $\mathrm{n}(\%)$ & $2,137(94.0)$ & $813(85.6)$ & $3,366(93.8)$ & $1,161(85.8)$ \\
\hline Low employment grade, $\mathrm{n}(\%)$ & $86(7.9)$ & $166(37.4)$ & $179(7.2)$ & $342(33.5)$ \\
\hline Current smoker, $\mathrm{n}(\%)$ & $200(8.8)$ & $110(11.7)$ & $194(5.6)$ & $58(4.5)$ \\
\hline Little or none physical activity, $\mathrm{n}(\%)$ & $1,633(72.2)$ & $783(83.3)$ & $2,478(69.2)$ & $1,038(76.9)$ \\
\hline High alcohol intake, n (\%) & $687(30.2)$ & $171(18.0)$ & $734(20.5)$ & $225(16.6)$ \\
\hline Cardio-metabolic condition, $\mathrm{n}(\%)^{\mathrm{b}}$ & $971(42.7)$ & $430(45.3)$ & $1666(56.7)$ & $672(60.3)$ \\
\hline
\end{tabular}






${ }^{b}$ Presence of any of the following cardio-metabolic conditions: diagnosed coronary heart disease, heart failure, stroke, diabetes, hypertension and/or obesity Abbreviations: HF-HRV = high frequency heart rate variability; LF-HRV = low frequency heart rate variability; RMSSD = root mean square of successive differences of normal-tonormal RR intervals; SDNN = standard deviation of all intervals between R waves with normal-to-normal conduction 
Table 2 Cross-sectional relationship of heart rate and HRV measures with depressive symptoms at phase 5 (1997-1999) and phase 9 (2007-2009) in individuals without antidepressant use

\begin{tabular}{|c|c|c|c|c|c|c|}
\hline & \multicolumn{3}{|c|}{ Baseline (Phase 5) } & \multicolumn{3}{|c|}{ Follow-up (Phase 9) } \\
\hline & $\mathrm{n}$ & $\beta(\mathrm{CI})$ & $p$ & $\mathrm{n}$ & $\beta(\mathrm{CI})$ & $p$ \\
\hline \multicolumn{7}{|c|}{ Heart rate } \\
\hline Men & 2224 & $0.01(-0.27$ to 0.26$)$ & 0.97 & 3335 & $0.18(-0.11$ to 0.47$)$ & 0.22 \\
\hline Women & 912 & $0.03(-0.31$ to 0.37$)$ & 0.86 & 1207 & $0.00(-0.36$ to 0.36$)$ & 0.99 \\
\hline \multicolumn{7}{|l|}{ SDNN } \\
\hline Men & 2224 & $-0.01(-0.02$ to 0.01$)$ & 0.26 & 3335 & $-0.01(-0.03$ to -0.001$)$ & 0.04 \\
\hline Women & 912 & $-0.01(-0.02$ to 0.01$)$ & 0.48 & 1207 & $-0.003(-0.02$ to 0.01$)$ & 0.70 \\
\hline \multicolumn{7}{|c|}{ LF-HRV } \\
\hline Men & 2224 & $-0.02(-0.04$ to 0.01$)$ & 0.20 & 3335 & $-0.03(-0.06$ to -0.01$)$ & 0.02 \\
\hline Women & 912 & $-0.01(-0.04$ to 0.03$)$ & 0.77 & 1207 & $-0.02(-0.05$ to 0.02$)$ & 0.42 \\
\hline \multicolumn{7}{|c|}{ HF-HRV } \\
\hline Men & 2224 & $-0.02(-0.04$ to 0.01$)$ & 0.24 & 3335 & $-0.04(-0.07$ to -0.001$)$ & 0.04 \\
\hline Women & 912 & $0.001(-0.04$ to 0.04$)$ & 0.96 & 1207 & $-0.01(-0.05$ to 0.03$)$ & 0.73 \\
\hline \multicolumn{7}{|l|}{ RMSSD } \\
\hline Men & 2224 & $-0.002(-0.02$ to 0.01$)$ & 0.82 & 3335 & $-0.01(-0.03$ to 0.01$)$ & 0.14 \\
\hline Women & 912 & $-0.002(-0.02$ to 0.02$)$ & 0.83 & 1207 & $-0.01(-0.03$ to 0.02$)$ & 0.67 \\
\hline
\end{tabular}

Abbreviations: $\mathrm{CI}=$ confidence interval; $\mathrm{HF}-\mathrm{HRV}=$ high frequency heart rate variability; LF$\mathrm{HRV}=$ low frequency heart rate variability; RMSSD = root mean square of successive differences of normal-to-normal RR intervals; SDNN = standard deviation of all intervals between $\mathrm{R}$ waves with normal-to-normal conduction 
Table 3 Prospective association between heart rate and HRV measures at phase 5 (19971999) and depressive symptoms at phase 9 (2007-2009)

\begin{tabular}{|c|c|c|c|c|}
\hline & $\mathrm{n}^{\mathrm{a}}$ & $\mathrm{OR}^{\mathrm{b}}$ & $95 \% \mathrm{CI}$ & $p$ \\
\hline \multicolumn{5}{|c|}{ Heart rate } \\
\hline Men & 1690 & 1.02 & $1.01-1.04$ & 0.011 \\
\hline Women & 644 & 1.03 & $1.00-1.05$ & 0.062 \\
\hline \multicolumn{5}{|l|}{ SDNN } \\
\hline Men & 1690 & 0.57 & $0.36-0.89$ & 0.013 \\
\hline Women & 644 & 0.62 & $0.33-1.18$ & 0.147 \\
\hline \multicolumn{5}{|l|}{ LF-HRV } \\
\hline Men & 1690 & 0.77 & $0.63-0.94$ & 0.010 \\
\hline Women & 644 & 0.80 & $0.59-1.08$ & 0.143 \\
\hline \multicolumn{5}{|l|}{ HF-HRV } \\
\hline Men & 1690 & 0.81 & $0.67-0.98$ & 0.031 \\
\hline Women & 644 & 0.90 & $0.70-1.16$ & 0.424 \\
\hline \multicolumn{5}{|l|}{ RMSSD } \\
\hline Men & 1690 & 0.65 & $0.46-0.92$ & 0.016 \\
\hline Women & 644 & 0.76 & $0.48-1.21$ & 0.255 \\
\hline \multicolumn{5}{|c|}{${ }^{\mathrm{a}}$ participants without depressive episode at phase 4 and 5 (GHQ $\geq 4$ or use of antidepressant } \\
\hline \multicolumn{5}{|c|}{${ }^{\mathrm{b}} \mathrm{OR}$ adjusted for age, ethnicity and depressive symptoms score at phase 5} \\
\hline \multicolumn{5}{|c|}{ Abbreviations: $\mathrm{CI}=$ confidence interval; HF-HRV = high frequency heart rate variability; LF- } \\
\hline \multicolumn{5}{|c|}{$\mathrm{HRV}=$ low frequency heart rate variability; RMSSD = root mean square of successive } \\
\hline \multicolumn{5}{|c|}{ differences of normal-to-normal RR intervals; SDNN = standard deviation of all intervals } \\
\hline
\end{tabular}


Table 4 Adjusted prospective association between heart rate and HRV at phase 5 (1997-1999) and depressive symptoms at phase $9^{\text {a }}$ (2007-2009)

in men

\begin{tabular}{lccccccccccccc}
\hline & Model 1 & $p$ & Model 2 & $p$ & Model 3 & $p$ & Model 4 & $p$ & Model 5 & $p$ & Model 6 & $p$ \\
& OR (95\% CI) & & OR (95\% CI) & & OR (95\% CI) & & OR (95\% CI) & & OR (95\% CI) & OR (95\% CI) \\
\hline Heart rate & $1.02(1.0-1.04)$ & 0.01 & $1.02(1.01-1.04)$ & 0.01 & $1.02(1.01-1.04)$ & 0.02 & $1.02(1.01-1.04)$ & 0.01 & $1.02(1.01-1.04)$ & 0.01 & $1.02(1.01-1.04)$ & 0.02 \\
SDNN & $0.57(0.36-.89)$ & 0.01 & $0.57(0.36-0.87)$ & 0.01 & $0.60(0.37-0.95)$ & 0.03 & $0.57(0.36-0.89)$ & 0.01 & $0.58(0.37-0.91)$ & 0.02 & $0.60(0.37-0.96)$ & 0.03 \\
LF-HRV & $0.77(0.63-0.94)$ & 0.01 & $0.77(0.63-0.94)$ & 0.01 & $0.78(0.64-0.96)$ & 0.02 & $0.77(0.63-0.94)$ & 0.01 & $0.78(0.63-0.95)$ & 0.01 & $0.78(0.63-0.97)$ & 0.02 \\
HF-HRV & $0.81(0.67-0.98)$ & 0.03 & $0.81(0.67-0.98)$ & 0.04 & $0.83(0.68-1.00)$ & 0.05 & $0.81(0.67-0.98)$ & 0.03 & $0.82(0.68-0.99)$ & 0.04 & $0.83(0.68-1.00)$ & 0.05 \\
RMSSD & $0.65(0.46-0.92)$ & 0.02 & $0.65(0.46-0.93)$ & 0.02 & $0.68(0.48-0.96)$ & 0.03 & $0.65(0.46-0.92)$ & 0.02 & $0.66(0.47-0.93)$ & 0.02 & $0.68(0.48-0.95)$ & 0.03 \\
\hline
\end{tabular}

${ }^{a}$ men without depressive episode at phase 4 and 5 (GHQ $\geq 4$ or use of antidepressant medication)

Abbreviations: $\mathrm{CI}=$ confidence interval; HF-HRV = high frequency heart rate variability; LF-HRV = low frequency heart rate variability; SDNN

$=$ standard deviation of all intervals between $\mathrm{R}$ waves with normal-to-normal conduction; RMSSD $=$ root mean square of successive differences of normal-to-normal RR intervals

Model 1 ( $\mathrm{n}=1690)$ : analysis adjusted for age, ethnicity and cognitive symptoms of depression score at phase $5 ;$ model $2(\mathrm{n}=1684)$ : model $1+$ socioeconomic status; model 3 ( $\mathrm{n}=1676)$ : model 1 + smoking, physical inactivity, high alcohol intake; model $4(\mathrm{n}=1690)$ : model $1+$ cardiometabolic condition (diagnosed coronary heart disease, heart failure, stroke, diabetes, hypertension and obesity); model 5 ( $\mathrm{n}=1690)$ : model $1+$ prescribed medication use; model $6(n=1670)$ : all covariates. 


\section{Figure title and legend}

Figure 1. Baseline Heart rate and HRV (1997-1999) and risk of incidence depressive symptoms 10 years later (2007-2009) in men and women without depressive episode at baseline. The figure shows that higher levels of heart rate and HRV measures are associated with higher and lower risk, respectively, of incidence depressive symptoms.

${ }^{\mathrm{b}} \mathrm{OR}$ adjusted for age, ethnicity and depressive symptoms score at phase 5 Abbreviations: HF-HRV = high frequency heart rate variability; LF-HRV = low frequency heart rate variability; RMSSD = root mean square of successive differences of normal-tonormal RR intervals; SDNN = standard deviation of all intervals between $\mathrm{R}$ waves with normal-to-normal conduction 